\title{
On some classical properties of doubly truncated mixture of burr XII and weibull distributions
}

\author{
Muhammad Daniyal ${ }^{1}$, Muhammad Aleem ${ }^{1}$, Tahir Nawaz ${ }^{2}$ \\ ${ }^{1}$ Department of Statistics, the Islamia University of Bahawalpur \\ ${ }^{2}$ Department of Statistics, G.C University Faisalabad
}

\section{Email address:}

muhamamd.daniyal@iub.edu.pk (M. Daniyal),draleemiub@hotmail.com (M. Aleem), tahir101@hotmail.com (T. Nawaz)

\section{To cite this article:}

Muhammad Daniyal, Muhammad Aleem, Tahir Nawaz. On Some Classical Properties of Doubly Truncated Mixture of Burr XII and Weibull Distributions. American Journal of Theoretical and Applied Statistics. Vol. 3, No. 2, 2014, pp. 55-59.

doi: $10.11648 /$ j.ajtas.20140302.15

\begin{abstract}
Limited work has been conducted on the doubly truncation for the mixture of different distributions. This paper is concerned with the doubly truncated mixture of Burr XII and Weibull distributions. In this paper, classical properties of the doubly truncated mixture of Burr XII and Weibull distributions have been derived. Cumulative distribution function, hazard rate, failure rate, inverse hazard function, odd function and the cumulative hazard function, rth moment about origin, moment generating function characteristic function, moments about origin and mean, mean and variance, measure of skewness and kurtosis have been discussed.
\end{abstract}

Keywords: Doubly Truncation, Mixture, Weibull and Burr XII Distributions, Characteristic Function

\section{Introduction}

Truncated distribution is the type of conditional distribution which is obtained by restricting the range of the other distribution. It arises in the practical situations where the ability to record the data or observation is limited to the values which lie above or below the given cut point.

The probability density function for the doubly truncated distribution is

$$
f_{D T}(x)=\frac{f(x)}{\int_{a}^{b} f(x) d x}, a<x<b
$$

Where $f(x)$ is the probability density function of the distribution

Truncation results in the data which are restricted above or below that result in the truncated sample. The truncation is applied to the particular distribution which yields in the new distribution and not belonging to that class of distribution. (B.R et al, 2000) in his study showed that the truncated distributions are utilized in many practical situations, especially in different industrial arrangements. Fuzzy truncated normal distribution with the applications of real life time data were completely discussed by H.Agahi and M.Ghezelayagh (2009). The estimation techniques of the maximum likelihood for the singly and doubly truncated normal distribution were firstly considered by Cohen, A. C (1950, 1991).

\section{Probability Density Function}

The probability density function for the doubly truncated mixture of Burr XII and Weibull distributions can be expressed in the following form.

$$
f_{D T}(x)=\frac{p_{1} c k s^{-c} x x^{c-1}\left[1+\left(\frac{x}{s}\right)^{c}\right]^{-k-1}+p_{2} \frac{\alpha}{\beta^{\alpha}} x^{\alpha-1} e^{-\left(\frac{x}{\beta}\right)^{\alpha}}}{\Lambda}
$$

Where

$$
\Lambda=\int_{a}^{b} p_{1} c k s{ }^{-c} x x^{c-1}\left[1+\left(\frac{x}{s}\right)^{c}\right]^{-k-1} d x+\int_{a}^{b} p_{2} \frac{\alpha}{\beta^{\alpha}} x^{\alpha-1} e^{-\left(\frac{x}{\beta}\right)^{\alpha}} d x
$$

Where $a<x<b, k, s, c, \alpha, \beta>0, p_{1}+p_{2}=1$

\section{Cumulative Distribution Function}

The cumulative distribution function for the doubly truncated mixture of burr XII and Weibull distributions is 
defined as

$$
F_{D T}(x)=\frac{\int_{a}^{x} f(x) d x}{\int_{a}^{b} f(x) d x}
$$

The final expression for the CDF is given below

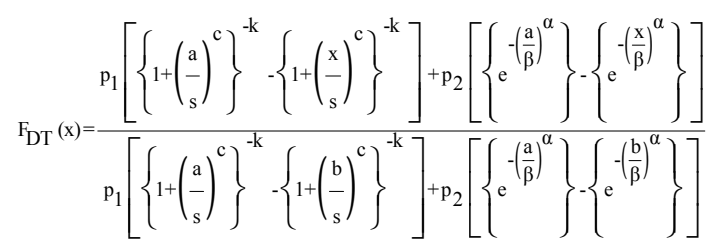

Where $a<x<b, k, s, c, \alpha, \beta>0, p_{1}+p_{2}=1$

\section{Reliability Function}

The reliability function is defined as follow

$$
R_{D T}(x)=1-F_{D T}(x)
$$

Putting equation (2.2) in (3.1), the required reliability function is defined as below
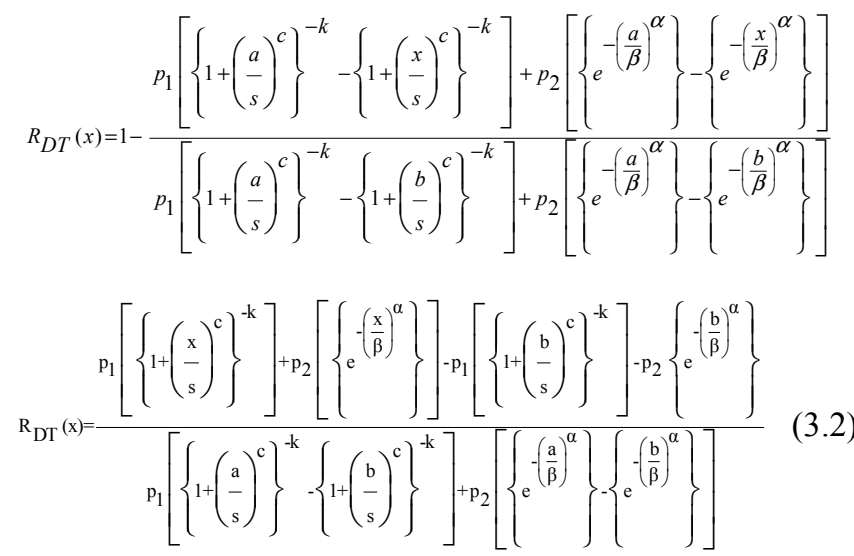

Where $a<x<b, k, s, c, \alpha, \beta>0, p_{1}+p_{2}=1$

\section{Hazard Function}

Hazard function is defined as the ratio of the probability density function and reliability function and is expressed in the following form

$$
h_{D T}(x)=\frac{f_{D T}(x)}{{ }^{R} D T}
$$

The hazard function can be obtained by putting (1.1) and (3.2) in (4.1) and the final expression is mentioned below

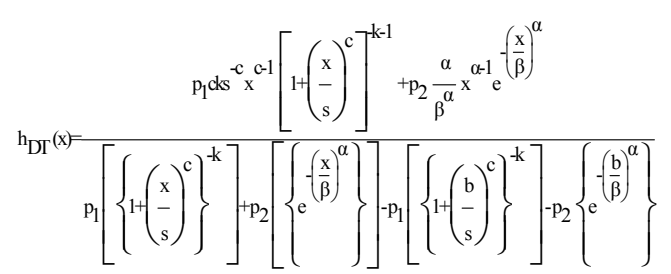

Where $a<x<b, k, s, c, \alpha, \beta>0, p_{1}+p_{2}=1$

\section{Cumulative Hazard Function}

The cumulative hazard function can be defined as

$$
\wedge_{D T}(x)=-\log R_{D T}(x)
$$

It is obtained by putting (3.2) in (5.1) and is expressed in the following form

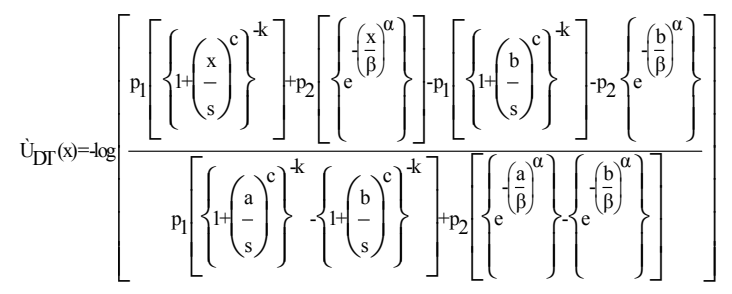

Where $a<x<b, k, s, c, \alpha, \beta>0, p_{1}+p_{2}=1$

\section{Reversed Hazard Function}

Reversed hazard rate can be defined as the ratio of the probability density function and the cumulative distribution function i.e

$$
r_{D T}(x)=\frac{f_{D T}(x)}{F_{D T}(x)}
$$

By putting (1.1) and (2.2) in (6.1) the expression obtained is as follow

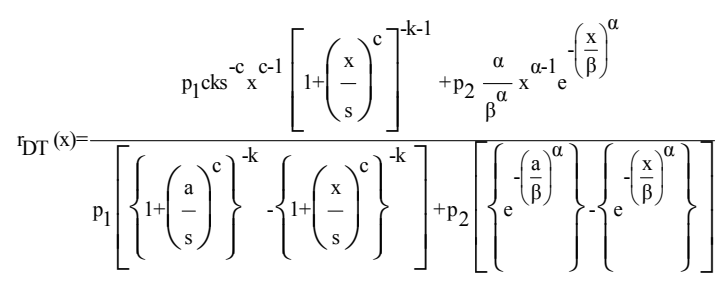

Where $a<x<b, k, s, c, \alpha, \beta>0, p_{1}+p_{2}=1$

\section{Odds Function}

The odds function denoted by $O(x)$ is the ratio of cumulative distribution function and the reliability function and has the following form:

$$
O_{D T}(x)=\frac{F_{D T}(x)}{R_{D T}(x)}
$$


The above expression can be obtained by putting (2.2) and (2.2) in (7.1) which as follow

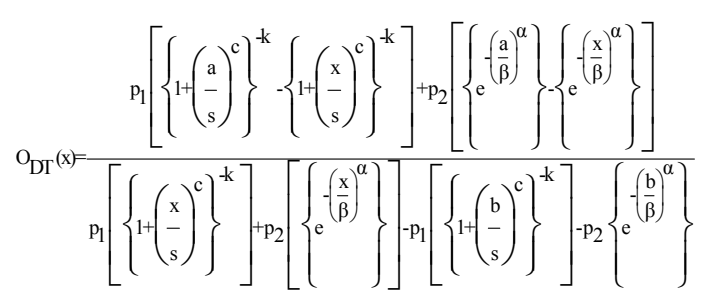

Where $a<x<b, k, s, c, \alpha, \beta>0, p_{1}+p_{2}=1$

\section{8. $\mathbf{R}^{\text {th }}$ Moment about Origin}

$\mathrm{r}^{\text {th }}$ moment for the real valued function can be defined as

$$
\begin{gathered}
\mu_{r}^{\prime}=E\left(x^{r}\right) \\
\mu_{r}^{\prime}=\int x^{r} f_{D T}(x) d x
\end{gathered}
$$

The final expression for the $\mathrm{r}^{\text {th }}$ moment about origin is as follow

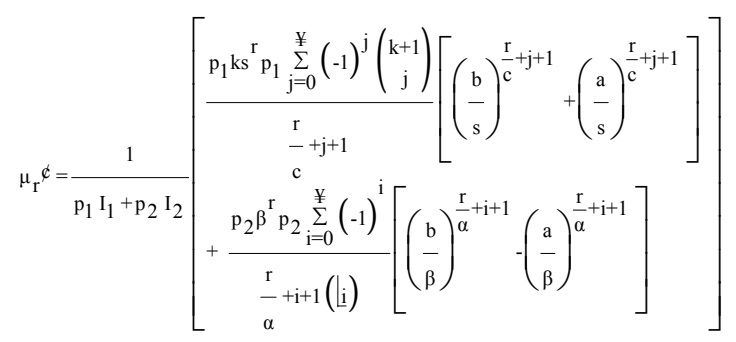

Where

$$
I_{1}=\left[\left\{1+\left(\frac{a}{s}\right)^{c}\right\}^{-k}-\left\{1+\left(\frac{b}{s}\right)^{c}\right\}^{-k}\right]
$$

and

$$
I_{2}=\left[\left\{e^{-\left(\frac{a}{\beta}\right)^{\alpha}}\right\}-\left\{e^{-\left(\frac{b}{\beta}\right)^{\alpha}}\right\}\right]
$$

\section{Raw Moments about Origin}

Putting $r=1,2,3$ and 4 in equation (8.1) the first four raw moments obtained are as follow

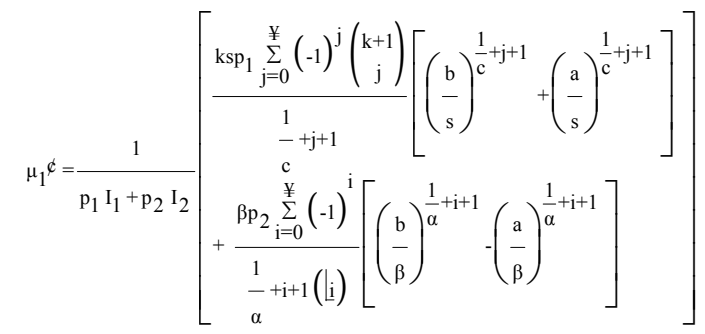

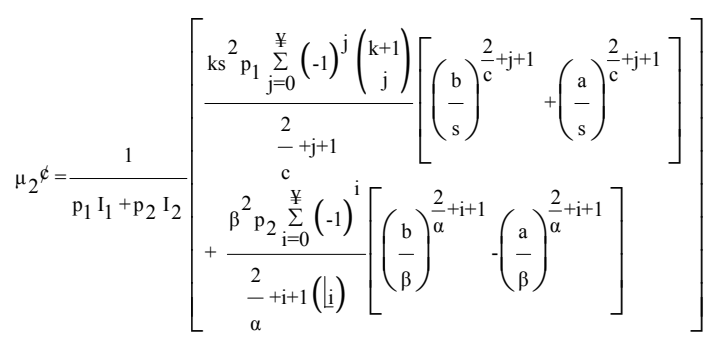

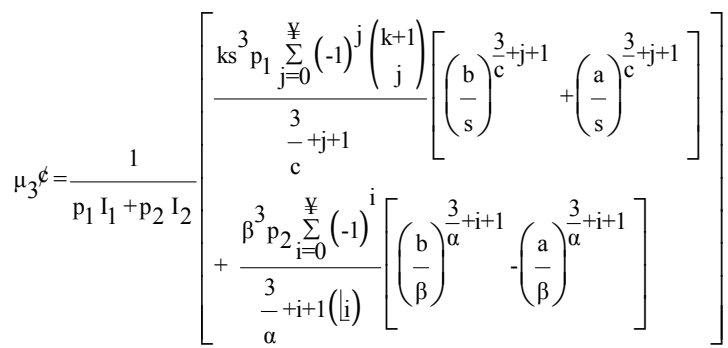

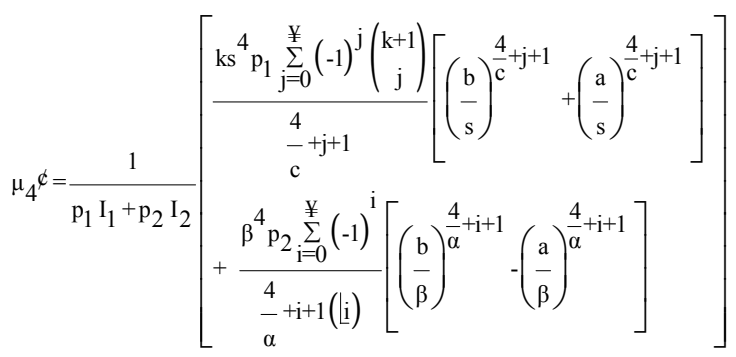

Where

$$
I_{1}=\left[\left\{1+\left(\frac{a}{s}\right)^{c}\right\}^{-k}-\left\{1+\left(\frac{b}{s}\right)^{c}\right\}^{-k}\right]
$$

And

$$
I_{2}=\left[\left\{e^{-\left(\frac{a}{\beta}\right)^{\alpha}}\right\}-\left\{e^{-\left(\frac{b}{\beta}\right)^{\alpha}}\right\}\right]
$$

\section{Moments about Mean}

$$
\begin{gathered}
\mu_{1}=0 \\
\mu_{2}=\mu_{2}^{\prime}-\left(\mu_{1}^{\prime}\right)^{2}=\text { Variance }
\end{gathered}
$$




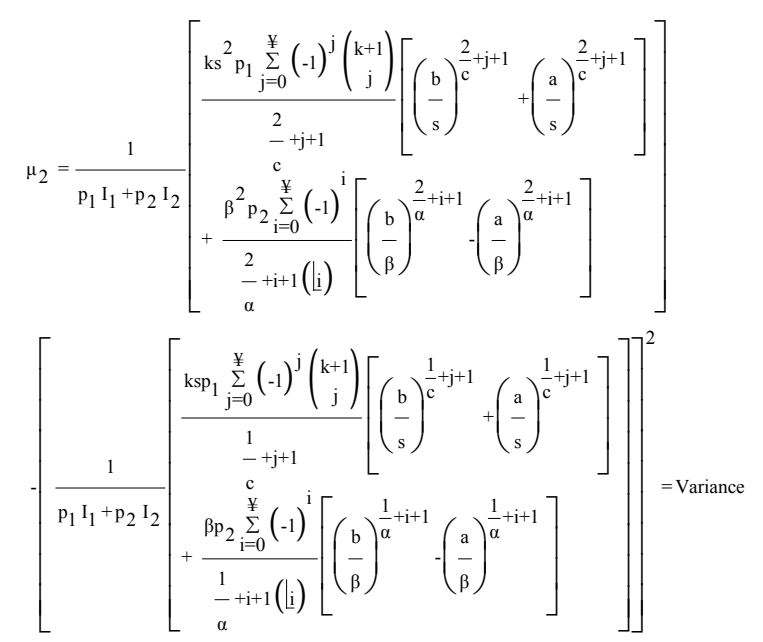

Where

$$
I_{1}=\left[\left\{1+\left(\frac{a}{s}\right)^{c}\right\}^{-k}-\left\{1+\left(\frac{b}{s}\right)^{c}\right\}^{-k}\right]
$$

And

$$
I_{2}=\left[\left\{e^{-\left(\frac{a}{\beta}\right)^{\alpha}}\right\}-\left\{e^{-\left(\frac{b}{\beta}\right)^{\alpha}}\right\}\right]
$$

The 3rd and 4th moments about mean can be calculated by putting $\mu_{1}^{\prime}, \mu_{2}^{\prime}, \mu_{3}^{\prime}$ and $\mu_{4}^{\prime}$ in the following equations respectively

$$
\begin{gathered}
\mu_{3}=\mu_{3}^{\prime}-3 \mu_{2}^{\prime} \mu_{1}^{\prime}+\left(\mu_{1}^{\prime}\right)^{3} \\
\mu_{4}=\mu_{4}^{\prime}-4 \mu_{1}^{\prime} \mu_{3}^{\prime}+6\left(\mu_{1}^{\prime}\right)^{2}\left(\mu_{2}^{\prime}\right)-3\left(\mu_{1}^{\prime}\right)^{4}
\end{gathered}
$$

\section{Measure of Skewness and Kurtosis}

Skewness is the measure of extent that the distribution leans to the one side of the mean and kurtosis is used to measure the flatness or peakedness of the probability curve.

Skewness and kurtosis are denoted by $\beta_{1}$ and $\beta_{2}$ respectively and is expressed in the following form

$$
\beta_{1}=\frac{\left(\mu_{3}\right)^{2}}{\left(\mu_{2}\right)^{3}}
$$

And

$$
\beta_{2}=\frac{\mu_{4}}{\left(\mu_{2}\right)^{2}}
$$

\section{Moment Generating Function}

The moment generating function can be defined as

$$
M_{x}(t)=E\left(e^{t x}\right)
$$

Where

$$
\begin{gathered}
E\left(e^{t x}\right)=\int e^{t x} f_{D T}(x) d x \\
f_{D T}(x)=\frac{p_{1} c k s x^{-c} c-1\left[1+\left(\frac{x}{-}\right)^{c}\right]^{-k-1}+p_{2} \frac{\alpha}{\beta} x^{\alpha-1} e^{-\left(\frac{x}{\beta}\right)^{\alpha}}}{p_{1}\left[\left\{1+\left(\frac{a}{s}\right)^{c}\right\}^{-k}-\left\{1+\left(\frac{b}{s}\right)^{c}\right\}^{-k}\right]+p_{2}\left[\left\{e^{-\left(\frac{a}{\beta}\right)^{\alpha}}\right\}-\left\{e^{-\left(\frac{b}{\beta}\right)^{\alpha}}\right\}\right]}
\end{gathered}
$$

The final expression for the moment generating function is as follow

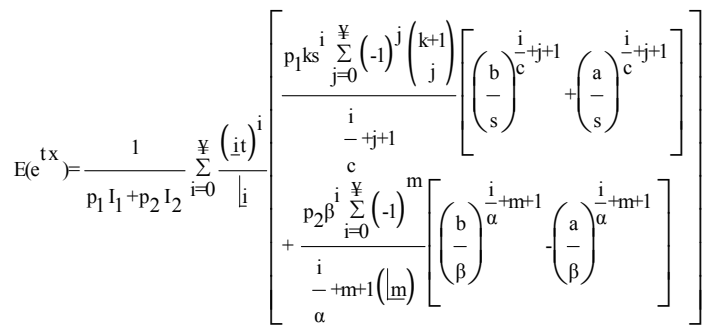

Where

$$
I_{1}=\left[\left\{1+\left(\frac{a}{s}\right)^{c}\right\}^{-k}-\left\{1+\left(\frac{b}{s}\right)^{c}\right\}^{-k}\right]
$$

And

$$
I_{2}=\left[\left\{e^{-\left(\frac{a}{\beta}\right)^{\alpha}}\right\}-\left\{e^{-\left(\frac{b}{\beta}\right)^{\alpha}}\right\}\right]
$$

\section{Characteristic Function}

Characteristic function is obtained by replacing $t$ in moment generating function with it and is expressed as below

$$
\varphi_{x}(t)=E\left(e^{i t x}\right)
$$

Its expression can be obtained by replacing $t$ with $i t$ in the expression of moment generating function (12.2) which is

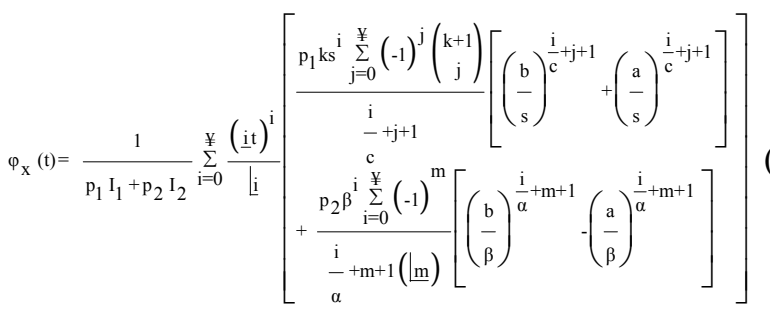


Where

$$
I_{1}=\left[\left\{1+\left(\frac{a}{s}\right)^{c}\right\}^{-k}-\left\{1+\left(\frac{b}{s}\right)^{c}\right\}^{-k}\right]
$$

\section{References}

[1] B.R. Cho, M.S. Govindaluri. (2002), Optimal Screening Limits in Multi-Stage Assemblies, International Journal Production Research 40, pp. 1993-2009.

And

$$
I_{2}=\left[\left\{e^{-\left(\frac{a}{\beta}\right)^{\alpha}}\right\}-\left\{e^{-\left(\frac{b}{\beta}\right)^{\alpha}}\right\}\right]
$$

[2] H.Agahi and M.Ghezelayagh. (2009), Fuzzy Truncated Normal Distribution with Applications, International Journal of Applied Mathematics and Computation, 1(3), pp170-181.

[3] Cohen, A. C. (1950), Estimating the mean and variance of normal populations from singly truncated and doubly truncated samples, Ann. Math. Statist., 21,pp 557-569.

[4] Cohen, A. C. (1991), Truncated and Censored Samples, Marcel Dekker, New York 